\title{
METABOLISMO, OXIDAÇÃO E IMPLICAÇÕES BIOLÓGICAS DO ÁCIDO DOCOSAHEXAENOICO EM DOENÇAS NEURODEGENERATIVAS
}

Patricia Postilione Appolinário, Priscilla Bento Mattos Cruz Derogis, Tatiana Harumi Yamaguti e Sayuri Miyamoto*

Departamento de Bioquímica, Instituto de Química, Universidade de São Paulo, Av. Prof. Lineu Prestes, 748, 05508-900

São Paulo - SP, Brasil

Recebido em 16/8/10; aceito em 20/4/11; publicado na web em 8/7/11

\begin{abstract}
METABOLISM, OXIDATION AND BIOLOGICAL IMPLICATIONS OF DOCOSAHEXAENOIC ACID IN NEURODEGENERATIVE DISEASES. Docosahexaenoic acid (C22:6, $n-3$, DHA) is a polyunsaturated fatty acid (PUFA) present in large concentrations in the brain and, due to the presence of six double bonds in its structure, is highly susceptible to oxidation by enzymes and reactive oxygen/nitrogen species. The peroxidation of PUFAs has been implicated in an increasing number of human disorders, including neurodegenerative diseases. Hence, a better understanding of the metabolism pathways of DHA should provide new insights about its role in neurodegenerative diseases. Here we review the main aspects related to DHA metabolism, as well as, the recent findings showing its association with neurodegenerative diseases.
\end{abstract}

Keywords: docosahexaenoic acid; metabolism; neurodegenerative diseases.

\section{INTRODUÇÃO}

Os lipídios constituem um grupo heterogêneo de compostos que têm papel crucial na célula, cuja característica comum é a solubilidade em solventes orgânicos e insolubilidade na água. As funções biológicas dos mesmos são tão diversas quanto a sua química. Sua importância pode ser notada pelo número crescente de estudos apontando o envolvimento de seus produtos de oxidação e de disfunções no metabolismo de lipídios em doenças crônico-degenerativas. ${ }^{1-4}$

Os ácidos graxos são ácidos carboxílicos com cadeias de hidrocarbonetos contendo de 4 a 36 carbonos, sendo encontrados principalmente esterificados a triglicerídios, colesterol e fosfolipídios constituintes das membranas celulares. Eles podem ser classificados em saturados (contendo apenas ligações simples), monoinsaturados (uma ligação dupla) ou poli-insaturados (duas ou mais ligações duplas). Os ácidos graxos poli-insaturados podem ser representados usando-se a abreviação estrutural que os identifica única e exclusivamente através do número de carbonos e de duplas ligações. Também podem ser denominados a partir da posição das ligações duplas, em relação ao grupamento metila terminal, utilizando-se para tanto a denominação $\omega$ ou $n,{ }^{5}$ sendo a denominação $n$ a recomendada pela IUPAC. ${ }^{6}$

Estudos mostram que os ácidos graxos poli-insaturados $n$-3 (ômega-3) e $n$-6 (ômega-6) têm efeitos distintos nas células, sendo o primeiro associado a efeitos benéficos e o segundo, a efeitos deletérios. ${ }^{7,8}$ Os ácidos linoleico (18:2n-6) e linolênico (18:3n-3) são os precursores dos ácidos graxos $n-6$ e $n$-3, respectivamente. São considerados essenciais por não serem sintetizados de novo pelos tecidos de vertebrados, sendo necessária a ingestão destes pela dieta. ${ }^{9}$

O ácido docosahexaenoico $(4,7,10,13,16,19-22: 6$, DHA) é um ácido graxo ômega-3 de 22 carbonos e 6 insaturações. Está enriquecido nas membranas neurais do córtex cerebral e na retina, ${ }^{10,11}$ onde 25-35\% se encontram esterificados em aminofosfolipídios como a fosfatidilserina, fosfatidiletanolamina e em plasmalogênios..$^{12,13} \mathrm{O}$ DHA constitui $>17 \%$ do peso total de ácidos graxos no cérebro

\footnotetext{
*e-mail: miyamoto@iq.usp.br
}

de ratos adultos e $>33 \%$ do total de ácidos graxos na retina. ${ }^{14,15} \mathrm{O}$ acréscimo do DHA no sistema nervoso central ocorre ativamente durante o período de desenvolvimento perinatal. ${ }^{12,16}$ Está envolvido no funcionamento da memória, ${ }^{17}$ na função excitável da membrana, ${ }^{18}$ biogênese e função de células fotorreceptoras, ${ }^{19}$ sinalização neuronal ${ }^{20}$ e neuroproteção. ${ }^{21,22}$

Neste artigo de revisão são abordados os principais aspectos relacionados ao metabolismo, à oxidação (enzimática e não enzimática) do DHA, bem como são mostrados e discutidos os aspectos recentes sobre as implicações deste ácido graxo em doenças neurodegenerativas.

\section{SÍNTESE E METABOLISMO DO DHA EM MAMÍFEROS}

As vias metabólicas de síntese dos ácidos graxos poli-insaturados (PUFAs) foram elucidadas por dois grupos de pesquisadores há mais de 40 anos (revisado em detalhes por Sprecher) ${ }^{23}$ Estes desenvolveram técnicas para sintetizar PUFAs marcados $\left(1-{ }^{14} \mathrm{C}\right)$, os quais foram administrados a ratos machos albinos. Os PUFAs dos tecidos foram isolados e saponificados, sendo o perfil de marcação desses compostos usado para propor suas vias de biossíntese..$^{24,25}$ Estabeleceu-se que o DHA pode ser sintetizado a partir do ácido $\alpha$-linolênico $(9,12,15-18: 3$, ALA) através de uma sequência de reações envolvendo dessaturações e alongamentos da cadeia carbônica. ${ }^{12,26-29}$ No entanto, estudos in vivo em humanos mostraram que $\sim 5 \%$ do ALA é convertido em EPA mas apenas $0,5 \%$ é convertido em DHA. Portanto, considera-se importante a ingestão de alimentos ou suplementos ricos em DHA. ${ }^{30}$

O fígado é considerado o principal local de síntese de DHA. ${ }^{28}$ Porém, um estudo realizado por Moore e colaboradores ${ }^{31}$ investigou se os próprios neurônios seriam capazes de alongar e dessaturar ácidos graxos essenciais ou estão dependentes do apoio de outras células cerebrais. Para tanto, culturas primárias de neurônios e astrócitos de rato foram incubadas com $\left[1-{ }^{14} \mathrm{C}\right] 18: 02 n-6\left[1-{ }^{14} \mathrm{C}\right] 20: 04 n-6,\left[1-{ }^{14} \mathrm{C}\right]$ 18:03 n-3, ou $\left[1-{ }^{14} \mathrm{C}\right]$ 20:05 $n$-3. Culturas neuronais dessaturaram de forma ineficaz os ácidos graxos a cada passo da via, produzindo principalmente produtos de alongamento dos precursores de 18- e de 
20 carbonos. ${ }^{31}$ Em contraste, os astrócitos alongaram e dessaturaram ativamente os precursores de 18 - e 20 carbonos. ${ }^{32} \mathrm{~A}$ maioria dos ácidos graxos de cadeia longa, formados por culturas de astrócitos, particularmente AA e DHA, foi liberada no líquido extracelular. Embora incapaz de sintetizar AA e DHA, culturas neuronais captaram esses ácidos graxos a partir do meio. ${ }^{31}$ Dessa forma, os astrócitos, que estão situados em estreito contato com os neurônios, parecem desempenhar um papel importante de fornecimento de DHA para os neurônios. ${ }^{12}$

A via de síntese do DHA (Figura 1) inicia-se com a ação da enzima $\Delta 6$-dessaturase a partir da dessaturação do ácido $\alpha$-linolênico a 18:4n-3 (cis-6,9,12,15-18:4) pela enzima (enzima que insere uma dupla ligação na posição 6). Em seguida, este ácido graxo tem sua cadeia carbônica alongada a 20:4n-3 (cis-8,11,14,17-20:4) pela enzima elongase, sendo subsequentemente convertido a 20:5n-3 (cis-5,8,11,14,1720:5, ácido eicosapentaenoico, EPA) pela $\Delta 5$-dessaturase e alongado a 22:5n-3 (cis-5,8,11,14,17-22:5) no retículo endoplasmático. ${ }^{12}$ Ainda neste mesmo compartimento celular, o ácido graxo 22:5n-3 sofre adição de 2 carbonos formando 24:5 (cis-9,12,15,18,21-24:5), o qual é convertido a 24:6 (cis-6,9,12,15,18,21-24:6) pela ação de uma $\Delta 6$-dessaturase. ${ }^{12}$ As enzimas $\Delta 5$ e $\Delta 6$-dessaturases de mamíferos foram identificadas e clonadas. ${ }^{33,34}$ Porém, a enzima $\Delta 4$-dessaturase, responsável pela síntese direta de DHA a partir de 22:5n-3, foi identificada apenas em microalgas. Deste modo, em mamíferos, o DHA é sintetizado por meio de etapas adicionais envolvendo reações de alongamento, dessaturação e posterior encurtamento da cadeia nos peroxissomos. ${ }^{12} \mathrm{Um}$ estudo utilizando fibroblastos de pacientes com defeitos na oxidação mitocondrial e peroxissomal de ácidos graxos, mostrou que peroxissomos e não mitocôndrias estão envolvidos na formação do DHA. ${ }^{35}$ Deste modo, a partir da formação de 24:6n-3, este é transferido para os peroxissomos onde é convertido a DHA através da remoção de dois carbonos da cadeia por $\beta$-oxidação. $\mathrm{O}$ DHA formado é transferido de volta ao retículo endoplasmático e rapidamente incorporado aos fosfolipídios de membrana por nova esterificação ou por reação de deacilação-reacilação. ${ }^{12,26,36}$

Os ácidos graxos de cadeia longa $n-6$ são biossintetizados a partir do ácido linoleico (18:2n-6) utilizando uma via análoga e o mesmo sistema enzimático dos ácidos graxos $n$-3 (Figura 2). ${ }^{12}$ É interessante notar que na maioria dos tecidos, o ácido graxo de cadeia longa mais comumente encontrado é o ácido araquidônico (20:4n-6; AA), no entanto o cérebro é particularmente enriquecido em DHA. Postulase que o acúmulo preferencial de DHA neste tecido provavelmente seja consequência de um processo de captação, síntese e esterificação bastante efetiva deste ácido graxo na célula neuronal. ${ }^{31}$

\section{Incorporação do DHA no cérebro}

O DHA da dieta ou sintetizado no fígado é transportado até o cérebro. Na circulação sanguínea o DHA pode ser transportado ligado à albumina ou a lipoproteínas. A albumina atravessa facilmente a barreira hematoencefálica, no entanto argumenta-se que a quantidade de ácidos graxos livres não seja suficientemente alta para fornecer quantidades suficientes de DHA para o cérebro e, portanto, ácidos graxos esterificados presentes em lipoproteínas seriam a principal fonte de DHA exógeno. ${ }^{28}$ Existem dois fatores que controlam o grau de incorporação do DHA no cérebro: a taxa de sua dissociação da albumina plasmática e a esterificação do DHA à coenzima A pela acil-CoA sintetase de cadeia longa. ${ }^{37,38}$

Independente da sua fonte, o DHA é rapidamente internalizado pelos neurônios e incorporado nos fosfolipídios das membranas plasmáticas sinápticas e nas vesículas sinápticas. ${ }^{22,39-41}$ Os mecanismos de absorção do DHA pelas células neurais continuam a ser elucidados, sendo também identificado o envolvimento de proteínas como a ácido graxo translocase e proteínas transportadoras de ácido graxo. ${ }^{12,42}$

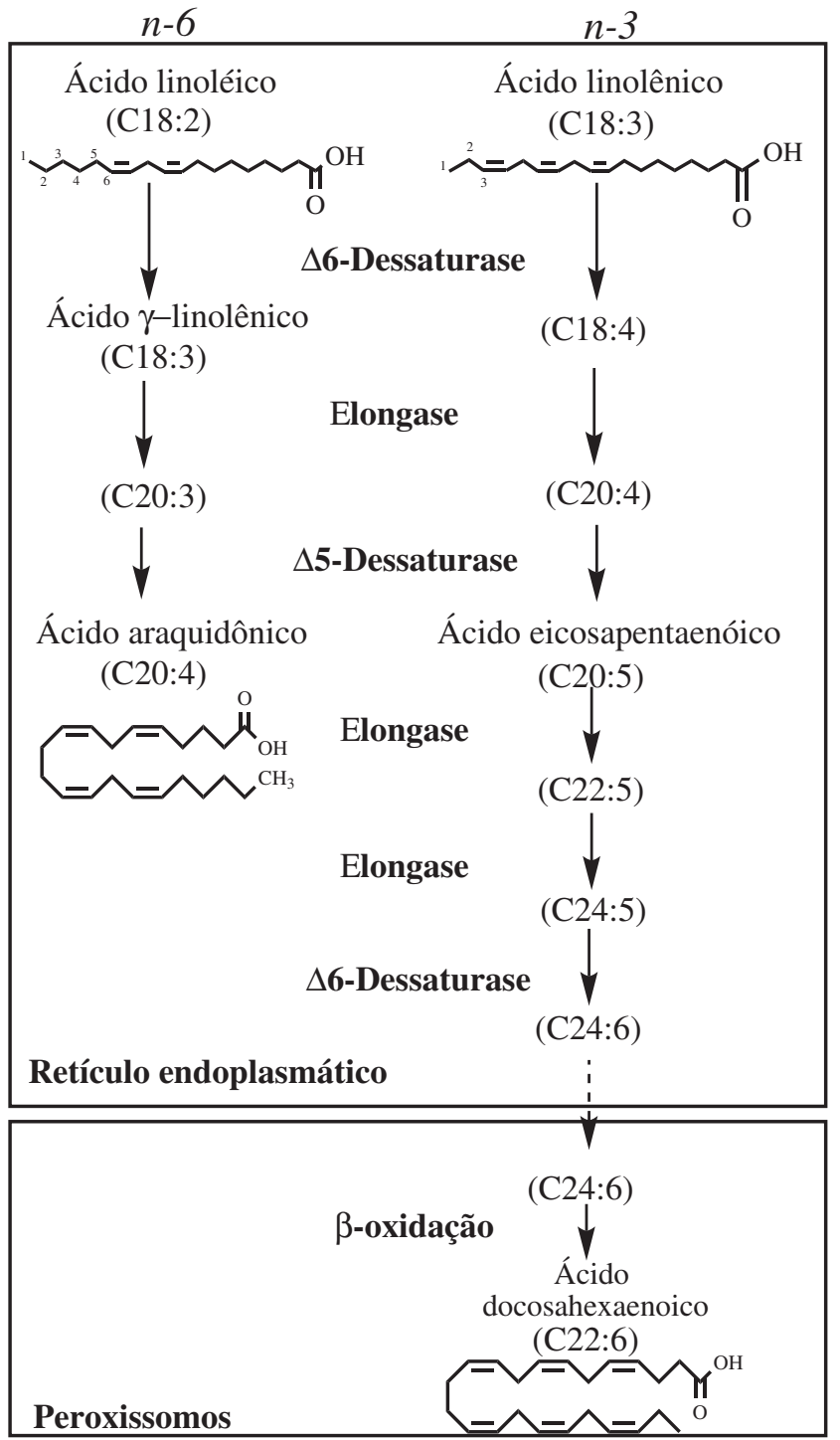

Figura 1. Esquema ilustrativo da via de síntese de ácidos graxos insaturados da série $n-3$ e $n-6$

Durante a síntese dos fosfolipídios, os ácidos graxos saturados, como o ácido palmítico (16:0) e o ácido esteárico (18:0) são acilados a posição sn-1 do glicerol 3-fosfato, enquanto os ácidos graxos poliinsaturados, como o DHA, são principalmente incorporados a posição $s n$-2. O DHA é incorporado, principalmente, em aminofosfolipídios, sendo a maior parte em fosfatidiletanolamina (PE) e em menor proporção na fosfatidilserina (PS). ${ }^{43,44}$ Experimentos realizados in vivo utilizando ratos acordados (recuperados da anestesia) mostraram que 2-8\% do DHA incorporado nos fosfolipídios do cérebro são substituídos diariamente em ratos adultos..$^{39}$ Neste experimento os ratos foram inicialmente anestesiados para implantação de um cateter na veia e artéria femoral direita e uma solução contendo o ácido graxo marcado radioativamente foi injetada após recuperação da anestesia. Medidas de radioatividade no plasma e no cérebro mostraram que essa reposição é feita pelo DHA não esterificado do plasma sanguíneo, sendo que em torno de $90 \%$ da radioatividade foi encontrada na fração lipídica contendo fosfolipídeos. ${ }^{39}$

Os DHAs não esterificados são rapidamente reincorporados a um lisofosfolipídio, através de ações de uma série de acil-CoA sintetases e aciltransferases com o consumo de ATP. ${ }^{45}$ Estudos mostram que a incorporação de PUFAs plasmáticos em fosfolipídios do 


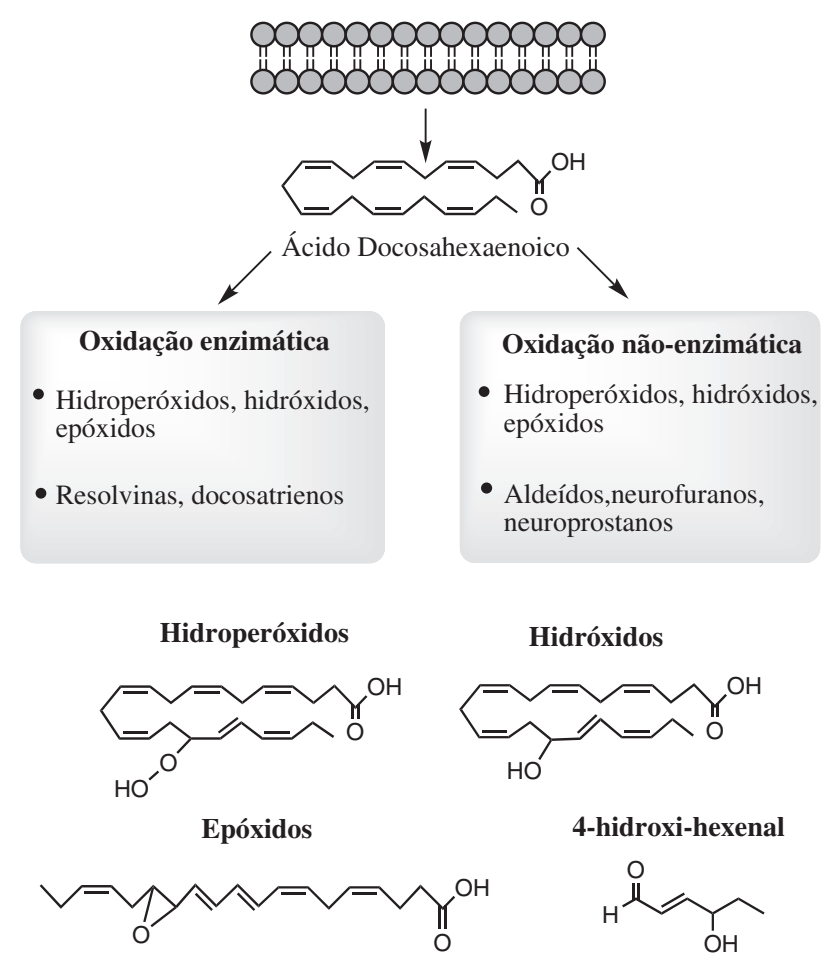

Docosatrienos<smiles>CC/C=C\CC(C)/C=C\C=C/C=C/CC/C=C/N=C/CCC(=O)O</smiles>

Resolvinas
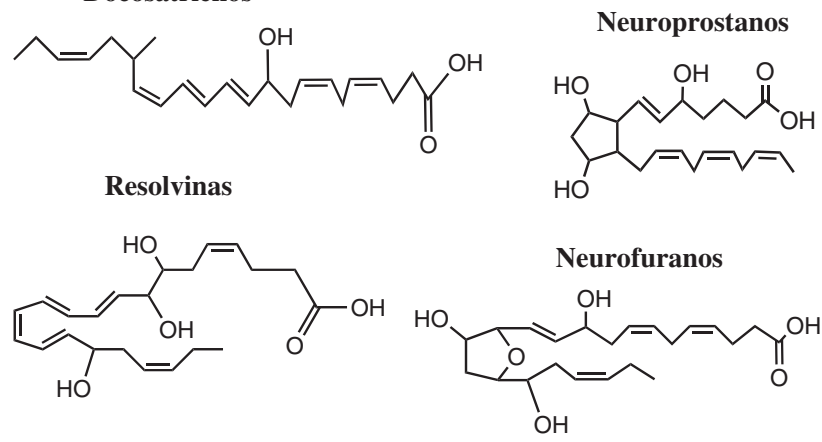

Figura 2. Representação esquemática dos produtos de oxidação do ácido docosa-hexaenoico por via enzimática e não enzimática. Estão representados hidroperóxidos, hidróxidos, epóxidos, resolvinas, docosatrienos, aldeídos, cetonas, neurofuranos e neuroprostanos

cérebro não é limitada por difusão pela barreira hematoencefálica, mas sim regulada pela conversão dependente de ATP dos PUFAs não esterificados dentro do cérebro a acil-CoA pela acil-CoA sintetase. ${ }^{39,46}$ Além da incorporação em fosfolipídeos, parte do DHA não esterificado captado pelos neurônios também é consumida através de um número de vias catabólicas, incluindo $\beta$-oxidação e conversão a eicosanoides ou docosanoides pelas ciclo-oxigenases (COX), lipooxigenases (LOX) ou citocromo P450 epoxigenases (EPOX) ${ }^{47,48} \mathrm{e}$ também por vias não enzimáticas mediadas por espécies reativas de oxigênio ou nitrogênio.

\section{OXIDAÇÃO DO DHA}

A oxidação dos ácidos graxos pode ser enzimática ou não enzimática. Estudos indicam que a oxidação enzimática gera compostos específicos com atividade anti-inflamatória, os quais podem estar envolvidos nos mecanismos de proteção mediados por DHA. ${ }^{49-51}$ Por outro lado, a oxidação não enzimática promovida por espécies reativas gera uma série de compostos muitos deles ainda pouco estudados (Figura 2).

\section{Oxidação enzimática}

A oxidação de ácidos graxos catalisada por LOX e COX é altamente regioespecífica e estereoespecífica. Essas enzimas são conhecidas pela geração de mediadores inflamatórios conhecidos como eicosanoides (prostaglandinas, leucotrienos e tromboxanos) a partir do ácido araquidônico. Em ambas, a enzima requer uma ativação inicial com a oxidação do átomo de ferro do seu centro ativo por pequenas quantidades de hidroperóxidos e, em seguida, a abstração do hidrogênio do grupo metilênico do ácido graxo, formando o radical centrado no carbono, que ao se combinar com $\mathrm{O}_{2}$ forma um radical peroxila. No mecanismo de catálise da COX, o radical peroxila sofre ciclização formando um endoperóxido que rapidamente se adiciona a mais uma molécula de oxigênio, formando um intermediário hidroperoxi endoperóxido. O mecanismo completo da COX envolve a redução do grupamento hidroperóxido deste endoperóxido pela atividade peroxidásica da enzima. No mecanismo de catálise da LOX, o radical peroxila é reduzido a um ânion peróxido e protonado formando, como produto final, hidroperóxidos. ${ }^{52}$ As vias de oxidação enzimática do DHA (n-3), assim como do ácido araquidônico ( $n-6)$, envolvem essas duas principais enzimas, além das EPOX.

Três isoformas de COX (COX-1, COX-2 e COX-3) estão presentes nos tecidos animais, incluindo o cérebro. ${ }^{48} \mathrm{COX}-1$ é expressa constitutivamente na grande maioria dos tecidos. COX-2 é induzível em resposta a uma variedade de estímulos agonistas, sendo o principal alvo de ação dos anti-inflamatórios não esteroidais. COX-3 é uma variante da COX-1 sensível ao acetaminofeno (N-acetil-paminofenol). O substrato preferencial para a COX-1 e COX-2 é o ácido araquidônico.

O ácido acetilsalicílico (AAS) além de ser um conhecido inibidor da formação de prostaglandinas pró-inflamatórias, induz a formação de mediadores lipídicos anti-inflamatórios. ${ }^{53} \mathrm{O}$ AAS modifica covalentemente o resíduo de serina próximo ao sítio ativo da enzima, através de uma reação de acetilação. A COX-1 perde a capacidade de oxidar o ácido araquidônico, enquanto que a COX-2 acetilada passa a converter o DHA em mediadores lipídicos, como resolvinas e protectinas. ${ }^{50,54}$

O tecido nervoso apresenta 3 isoformas de LOX (5-LOX, 12LOX e 15-LOX) capazes de oxidar DHA gerando hidroperóxidos específicos. ${ }^{55} \mathrm{~A}$ nomenclatura das diferentes isoformas da LOX é baseada na posição que a mesma oxida o ácido araquidônico. O DHA pode ser convertido pela 5-LOX (enzima responsável pela produção de leucotrienos a partir do ácido araquidônico) em 7-hidroperoxi-DHA e sua forma reduzida 7-hidroxi-DHA. Em outro trabalho, LOX de plaquetas mostraram ser capazes de oxidar DHA a 11- e 14-hidroperoxi (ou hidroxi)-DHA. Mais recentemente, uma série de produtos di-hidroxilados e tri-hidroxilados de DHA foram caracterizadas, sendo os compostos mais bioativos denominados de resolvinas (compostos capazes de promover a resolução da inflamação), ${ }^{56}$ e protectinas (compostos com alto potencial antiinflamatório contendo trienos conjugados ou docosatrienos).$^{54} \mathrm{~A}$ neuroprotectina D1 apresenta ações neuroprotetoras, tais como indução de proteínas Bcl-2 antiapoptóticas e inibição da expressão de proteínas Bcl-2 pró-apoptóticas. ${ }^{57}$

Estudos realizados com células da glia, sangue total e leucócitos humanos indicam que a biossíntese dos mediadores protetores do DHA envolve a ação inicial da fosfolipase A2, que libera o ácido graxo. Este por sua vez, é convertido a ácido 17S-hidro(pero)xi-DHA ou ácido 17R-hidro(pero)xi-DHA, pela 15-LOX ou pela COX-2 acetilada, respectivamente. Estes intermediários sofrem uma sequência de reações resultando na formação de resolvinas da série D ou resolvinas da série $\mathrm{D}$ associadas à aspirina..$^{50,54}$ 


\section{Oxidação não enzimática}

O DHA é um ácido graxo altamente insaturado (possui 6 insaturações), consequentemente, susceptível à oxidação mediada por espécies reativas. ${ }^{58,59}$ Várias evidências apontam para envolvimento de espécies reativas de oxigênio e/ou nitrogênio na patogênese de doenças neurodegenerativas. ${ }^{60}$ Deste modo, lipídios poli-insaturados, em particular o DHA, são alvos bastante susceptíveis de oxidação, gerando neste processo uma série de produtos oxidados, entre eles, hidroperóxidos (LOOH). ${ }^{59,61,62}$ Sendo mais polares, os LOOHs modificam a estrutura e a função das membranas e podem ser deletérios para as células. $^{62}$

Os LOOH são os produtos primários de um processo autocatalítico de oxidação conhecido como peroxidação lipídica (Figura 3). Esse processo se inicia pelo ataque a um lipídio por qualquer espécie que tenha reatividade suficiente para abstrair um átomo de hidrogênio de um grupo metileno (- $\left.\mathrm{CH}_{2}-\right)$. Nesta fase, chamada de etapa de iniciação, tem-se a formação de um radical centrado no carbono metilênico $\left(-{ }^{\bullet} \mathrm{CH}-\right)$ pela retirada do seu $\mathrm{H}^{\bullet}$, provocando um desemparelhamento de elétrons neste mesmo carbono. O destino mais provável deste novo radical formado será combinar-se com o $\mathrm{O}_{2}$ produzindo novos radicais, ainda mais reativos. Nesta fase de propagação da peroxidação, novos radicais são formados pela combinação do radical de carbono com o $\mathrm{O}_{2}$, os radicais peroxil ou radical peróxido (LOO*), os quais reagirão com outros lipídeos adjacentes. É nesta fase que ocorrem reações em cadeia e a propagação da peroxidação lipídica. Esta combinação do radical peroxila com um átomo de hidrogênio abstraído de outra molécula gera hidroperóxidos de lipídeos (LOOH) ou apenas peróxidos. Íons de metais livres $\left(\mathrm{M}^{\mathrm{n}+}\right)$ também são capazes de promover a oxidação lipídica e a continuidade da reação de propagação pela quebra dos $\mathrm{LOOH}$ pré-formados, gerando LOO* e/ou radical alcoxila (LO*). A decomposição de $\mathrm{LOOH}$ promovida por metais reduzidos $\left(\mathrm{M}^{\mathrm{n}+}\right)$ gera $\mathrm{LO}^{*}$, enquanto os na forma oxidada $\left(\mathrm{M}^{\mathrm{n}+1}\right)$, produzem LOO ${ }^{*}{ }^{63}$ A cadeia de terminação efetivamente ocorre apenas quando há a reação de dois radicais peroxilas, formando carbonilas, alcoóis e oxigênio singlete, uma espécie excitada de $\mathrm{O}_{2}$ com alta capacidade oxidante. ${ }^{64-66}$

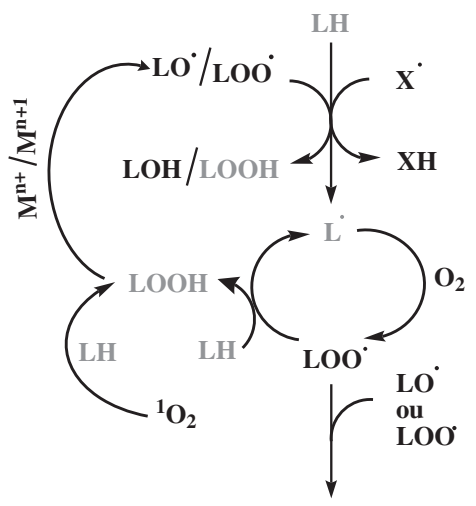

Produtos

Figura 3. Esquema do processo de peroxidação lipídica e da catálise mediada por metais $\left(M^{n+}\right.$ ou $\left.M^{n+1}\right)$. $X^{*}$ é qualquer radical capaz de abstrair um átomo de hidrogênio do lipídeo $(L H)$. $L \cdot$ é um radical centrado no carbono metilênico. $\mathrm{LOO}^{*}$ e $\mathrm{LO}^{*}$ são radicais peroxila e alcoxila, respectivamente

A formação de hidroperóxidos de DHA também pode ocorrer pela reação direta do ácido graxo com o oxigênio singlete $\left({ }^{1} \mathrm{O}_{2}\right)$, uma espécie excitada do oxigênio triplete. $\mathrm{O}^{1} \mathrm{O}_{2}$ adiciona-se a duplas ligações através de uma reação do tipo ene, produzindo hidroperóxidos. Neste caso, pode-se observar a formação de hidroperóxidos em posições específicas, além daqueles formados por reações envol- vendo radicais livres, ${ }^{67-69}$ A oxidação do DHA produz hidroperóxidos (HpDHA) como produto primário de oxidação. A oxidação mediada por radicais livres leva à formação de 10 mono-hidroperóxidos, 4-, 7-, 8-, 10-, 11-, 13-, 14- e 16-, 17 e 20-HpDHA, todos contendo dienos conjugados (Figura 4). Em contraste, a foto-oxidação do DHA leva à formação de 12 isômeros: os mesmos 10 formados pela oxidação mediada por radicais livres mais os isômeros 5- e 19- HpDHA que não apresentam dienos conjugados. ${ }^{59}$

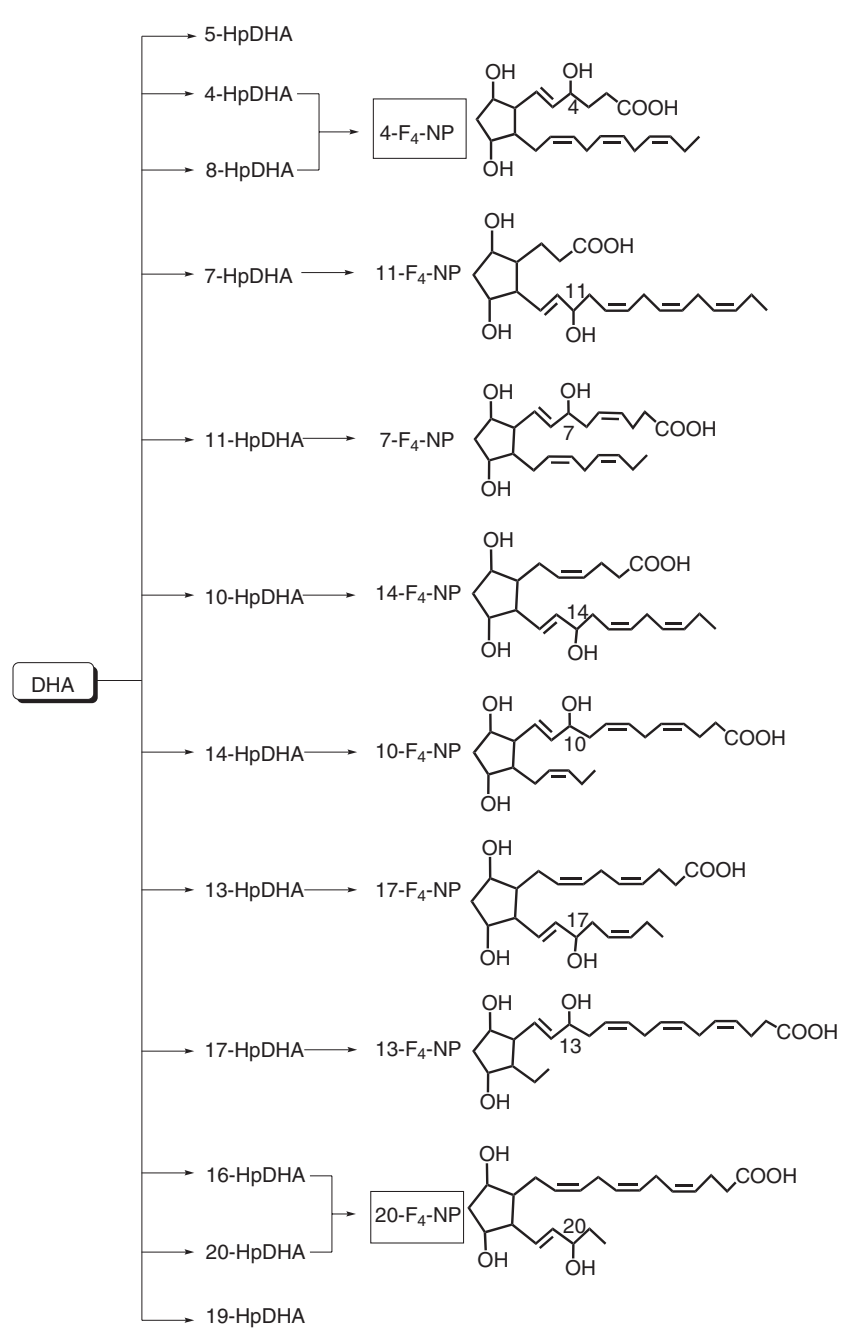

Figura 4. Esquema representativo dos hidroperóxidos derivados do DHA (4-, 7-, 8-, 10-, 11-, 13-, 14- e 16-, 17 e 20-HpDHA) e dos neuroprostanos identificados in vitro e in vivo ( 4- $F_{4}-N P, 11-F_{4}-N P, 7-F_{4}-N P\left(n P F_{4 a}-V\right), 14-F_{4}^{-}$ $N P, 10-F_{4}-N P, 17-F_{4}-N P, 13-F_{4}-N P, 20-F_{4}-N P$ ). A peroxidação do DHA leva à formação de oito possíveis grupos regioisoméricos de neuroprostanos, sendo que há a hipótese de que as séries 4 e 20- $F_{4}$-NPs são geradas em maior quantidade. Adaptado da ref. 61

Além dos hidroperóxidos, a oxidação do DHA gera uma série de produtos secundários como, por exemplo, os neuroprostanos (NP) ${ }^{61}$ neurofuranos, ${ }^{70}$ neurocetais ${ }^{71}$ e aldeídos de cadeia curta. ${ }^{72}$ Neuroprostanos, neurofuranos e neurocetais são produtos formados por reações de ciclização envolvendo radicais peroxila, enquanto os aldeídos de cadeia curta são formados por meio de quebra da cadeia do ácido graxo. A formação desses produtos tem sido revelada em amostras de cérebro, inclusive humano; além disso, estudos sugerem que esses produtos podem servir como bons marcadores da injúria oxidativa em tecidos enriquecidos em DHA, como o cérebro. ${ }^{73}$

Os NP são compostos análogos aos isoprostanos (IsoP), gerados 
pela via enzimática de oxidação do AA ${ }^{61} \mathrm{~A}$ principal classe de neuroprostanos identificada in vitro e in vivo contém anéis prostano do tipo $\mathrm{F}$ e são conhecidas como $\mathrm{F}_{4}-\mathrm{NPs}$. A peroxidação do DHA leva à formação de 8 possíveis grupos regioisoméricos, sendo que há a hipótese de que as séries 4 e 20-NPs são geradas em maior quantidade (Figura 4). ${ }^{61,74,75}$

Os neurofuranos são isofuranos caracterizados por um anel tetra-hidrofurano substituído. Eles são preferencialmente formados sob condições de alta tensão de oxigênio. ${ }^{70}$ Neurofuranos já foram detectados em níveis elevados no córtex cerebral em ratos de um modelo de doença de Alzheimer. ${ }^{76}$

A peroxidação dos ácidos graxos poli-insaturados leva também à formação de uma série de aldeídos citotóxicos e genotóxicos. ${ }^{77}$ Os aldeídos são relativamente estáveis e, consequentemente, capazes de se difundir dentro ou fora da célula e atacar células distantes do local onde foi iniciado o processo oxidativo. Aldeídos derivados da peroxidação do DHA têm sido descritos como compostos que apresentam um papel chave na patogênese das doenças neurodegenerativas e seus metabólitos são potenciais parâmetros de lipoperoxidação. ${ }^{78}$

Múltiplos aldeídos são derivados da peroxidação do DHA, acroleína, neurocetais, 4-hidroxi-trans-2-cis-6-nonadienal, e 4-hidroxi-trans-2-hexenal (HHE). O HHE é descrito na literatura como o principal produto de peroxidação in vitro do DHA. Apesar da possibilidade de ser produto de outros ácidos graxos ômega-3, no cérebro o DHA está presente em uma proporção 30 vezes maior que os demais e, por isso, o HHE é utilizado como biomarcador da oxidação do DHA.

\section{DHA E DOENÇAS NEURODEGENERATIVAS}

Doenças neurodegenerativas, como a doença de Alzheimer (AD), de Parkinson (PD) e a Esclerose Lateral Amiotrófica (ALS) são caracterizadas pela perda de células neuronais específicas e têm como mecanismo comum a presença de agregados proteicos. ${ }^{79} \mathrm{Com}$ relação à forma, há vários tipos de agregados, incluindo agregados do tipo amorfo e do tipo amiloide, sendo este último o mais característico. ${ }^{79}$ Os agregados do tipo amiloide caracterizam-se pela presença de fibrilas constituídas pelo empilhamento de folhas $\beta$ paralelas. A presença dessas folhas $\beta$ foi demonstrada para os agregados proteicos encontrados em AD e PD constituídos, respectivamente, de peptídeos $\beta$-amiloide e de $\alpha$-sinucleína. Até o momento não se sabe ao certo os mecanismos que disparam essa agregação, porém estudos recentes têm sugerido o envolvimento de fatores como o estresse oxidativo e também de lipídeos e seus produtos de oxidação nesse processo. ${ }^{80,81}$

Com relação aos lipídeos, alguns estudos têm mostrado uma diminuição no conteúdo de DHA em pacientes com AD. ${ }^{57,82}$ Dentre estes estudos, Lukiw e colaboradores ${ }^{57}$ observaram uma redução significativa de DHA em regiões específicas do cérebro, como o hipocampo, mantendo-se inalterado em outras regiões, como córtex e tálamo. Porém, não está claro se este declínio no conteúdo de DHA é um resultado das mudanças no metabolismo do ácido graxo ou pela modificação oxidativa do DHA. ${ }^{83}$ Como descrito anteriormente, o DHA é bastante susceptível à oxidação e pode ser oxidado tanto enzimática- como não enzimaticamente e estes processos podem ser responsáveis pelo consumo de DHA.

Outra característica comum a doenças neurodegenerativas é sua associação com a peroxidação lipídica., ${ }^{284-86}$ Porém, a interpretação de experimentos que investigam a contribuição de produtos da peroxidação lipídica para patogênese de doenças é limitada pela falta de especificidade bioquímica. ${ }^{2}$ Uma classe bastante estudada de produtos reativos é a dos aldeídos. Destes, os mais estudados são os gerados pela peroxidação do ácido araquidônico, como o 4-hidroxi2-nonenal e o 4-hidroxi-2-hexenal, um produto da peroxidação do
DHA. ${ }^{77,80}$ Embora as consequências fisiopatológicas da superprodução de 4-hidroxi-2-nonenal e 4-hidroxi-2-hexenal tenham sido destaque em diversos estudos, deve-se salientar que estes aldeídos reativos também são gerados em baixos níveis em todas as células e parecem ter um papel normal na sinalização fisiológica. ${ }^{87}$ Recentemente, outra classe de produtos de peroxidação lipídica reativos quimicamente foi identificada: $\alpha$-cetoaldeído isocetais, provenientes do AA e neurocetais provenientes do DHA. Estes cetoaldeídos são muito mais reativos que 4-hidroxi-2-nonenal ou 4-hidroxi-2-hexenal, ${ }^{88}$ sendo capazes de modificar uma série de biomoléculas, incluindo proteínas e ácidos nucleicos. Acredita-se que essas modificações tenham papel importante no desenvolvimento das doenças neurodegenerativas e sua quantificação tem sido utilizada como parâmetro para avaliar o dano oxidativo.

\section{Doença de Alzheimer (AD)}

A Doença de Alzheimer (AD) é uma desordem neurodegenerativa progressiva caracterizada pelo acúmulo de peptídeos amiloidogênicos $A \beta$ gerados pelo processamento da proteína precursora amiloide (APP) pela $\beta$ - e $\gamma$-secretase. ${ }^{89,90} \mathrm{Na} A D$, uma terceira protease associada à membrana, $\gamma$-secretase, cliva a APP dentro da porção transmembrana da proteína. Esta clivagem combinada com a clivagem extracelular da APP pela $\beta$-secretase, que parece ser a enzima limitante nesta via degenerativa, libera o peptídeo tóxico $A \beta$ na forma característica de 40 ou 42 aminoácidos. ${ }^{91,92}$ A liberação de fragmentos tóxicos de $\mathrm{A} \beta$ leva à formação inicial de agregados diméricos $\mathrm{e}$ oligoméricos solúveis. ${ }^{89}$ Uma agregação adicional leva à formação de complexos macromoleculares insolúveis que se depositam no meio extracelular do parênquima do cérebro para formar placas amiloides, um biomarcador patológico chave da AD. A combinação dos efeitos tóxicos de ambos, os agregados iniciais oligoméricos e o depósito de placas $A \beta$ insolúveis, leva a um rompimento neuronal e a uma morte celular eventual, que por sua vez é responsável pelo declínio cognitivo irreversível encontrado na AD..$^{89,90}$

A riqueza dos dados coletados a partir de ensaios in vitro, em cultura celular e modelos de animais transgênicos para AD suporta uma associação direta de PUFAs ômega-3, principalmente DHA, com o processamento amiloide no cérebro. PUFAs ômega-3 como componentes integrais de membrana podem agir para alterar o processamento amiloidogênico de várias maneiras distintas incluindo: a) facilitar a interação de $\alpha$-secretase com APP para produzir fragmentos não tóxicos e evitar a formação de $A \beta$; b) proteger a sequência de reconhecimento e sítio de clivagem intramembrana essencial para $\gamma$-secretase; c) servir como um local dissipador para os radicais livres que reduzem o aumento da atividade enzimática $\gamma$-secretase, que pode ser induzida por danos causados pelos radicais livres para o complexo de proteínas, o que é importante para a regulação da função normal $\gamma$-secretase e, d) inibir diretamente a fibrilação e formação de espécies oligoméricas de $\mathrm{A} \beta .^{89}$

Muitos estudos epidemiológicos mostraram uma relação inversa entre ingestão de ômega-3 e incidência de $\mathrm{AD}$, considerando que estudos clínicos encontraram pouco ou nenhum efeito pronunciado no estágio inicial da doença. Esses dados indicam que o DHA pode ter um efeito mais eficaz para prevenção do que para o tratamento dessa doença. ${ }^{90}$

Estudos com cultura celular demonstraram consistentemente uma redução de $20 \%$ da produção de $\mathrm{A} \beta$ depois do tratamento com o DHA. ${ }^{89,93,94}$ As atividades de ambas $\beta$ - e $\gamma$-secretase estavam diminuídas quando essas culturas celulares foram tratadas com DHA, o que está de acordo com os níveis reduzidos de $\mathrm{A} \beta{ }^{89,90}$ Esses efeitos parecem estar diretamente relacionados com a composição de $l i$ pid rafts na membrana, sendo uma das propostas de um grupo de 
pesquisadores (Grim e colaboradores) que o DHA reduz a geração de $\mathrm{A} \beta$ deslocando o colesterol para fora dos lipids rafts, reduzindo assim, a atividade enzimática responsável pelo processamento amiloidogênico da APP.90

\section{Doença de Parkinson (PD)}

A Doença de Parkinson (PD) é a desordem de movimento mais comum, afetando mais que $1 \%$ da população acima de 65 anos de idade. ${ }^{95}$ A maior parte dos casos ocorre esporadicamente com o avanço da idade, sendo um importante fator de risco. ${ }^{95}$ É caracterizada pela perda progressiva de neurônios dopaminérgicos e a deposição de corpos de inclusão intracelular na forma de corpos de Lewy e neuritos de Lewy. ${ }^{95-97} \mathrm{O}$ principal componente proteico desses depósitos é a $\alpha$-sinucleína. ${ }^{97} \mathrm{~A} \alpha$-sinucleína é uma proteína desdobrada caracterizada por sete repetições (KTKEGV) na região $\mathrm{N}$-terminal; por uma região central hidrofóbica formada por resíduos 61-65 ( componente não $\beta$-amiloide); e por trechos acídicos na parte C-terminal. ${ }^{98}$

Alguns estudos reportam que $\alpha$-sinucleína pode interagir com ácidos graxos poli-insaturados, cuja interação promoveria a oligomerização da proteína. ${ }^{95,96,98,99}$ Os níveis de DHA mostraram-se elevados nas áreas do cérebro contendo $\alpha$-sinucleína em pacientes com PD, o que atraiu o interesse no estudo da interação entre DHA e $\alpha$-sinucleína. ${ }^{98,100}$ De Francheschi e colaboradores ${ }^{98}$ mostraram $^{2}$ que $\alpha$-sinucleína interage com o DHA adquirindo uma conformação $\alpha$-helicoidal e o estado físico do lipídio torna-se alterado. Especificamente, métodos biofísicos e microscopia eletrônica indicaram que o DHA forma gotículas de óleo na presença da $\alpha$-sinucleína. Experimentos de proteólise limitada mostraram que, quando a proteína está ligada a essas gotículas de óleo, inicialmente é clivada na região de 89-102, sugerindo que este segmento da cadeia é suficientemente flexível para ser desdobrado ou protease-sensível. Eventos proteolíticos subsequentes produzem fragmentos correspondentes aos primeiros 70-80 resíduos que ficam estruturados e mostram uma elevada afinidade pelos lipídios. O fato de que uma região da cadeia polipeptídica permanece acessível a proteases, quando interage com os lipídios, sugere que esta região poderia estar envolvida em outras interações, justificando a propensão ambivalente de $\alpha$-sinucleína para dobrar ou agregar na presença de DHA. Outro grupo de pesquisadores (Broersen e colaboradores) também buscou explicar a interação entre $\alpha$-sinucleína e DHA. Mostraram que a $\alpha$-sinucleína faz com que o DHA esteja na forma de um polímero solúvel em vez de uma forma micelar. Após a interação com DHA, a $\alpha$-sinucleína que normalmente está desestruturada, rapidamente adota uma conformação $\alpha$-helicoidal. A exposição prolongada ao DHA, no entanto, gradualmente converte a $\alpha$-sinucleína em fibras do tipo amiloide. ${ }^{95}$

Assim como para doença de Alzheimer, várias linhas de evidência sugerem um papel importante para o estresse oxidativo e a PD. ${ }^{101}$ Neurônios dopaminérgicos (DA) são particularmente propensos ao estresse oxidativo, devido ao metabolismo dos DA, e auto-oxidação combinada com o aumento de ferro, níveis diminuídos de glutationa total e produção de ROS induzida pela inibição do complexo I mitocondrial induzida no sistema nervoso que pode levar à morte celular, por exceder a capacidade oxidativa na região das células contendo DA. ${ }^{101}$

\section{Esclerose Lateral Amiotrófica (ELA)}

É uma doença neurodegenerativa progressiva. ${ }^{102}$ É geralmente descrita como uma doença multifatorial, estando envolvidos estresse oxidativo, dano mitocondrial, excitotoxicidade, agregação de proteínas, diminuição do transporte axonal e morte dos neurônios motores. ${ }^{103}$ Apesar da doença se apresentar, na maioria dos casos, na sua forma esporádica, $10 \%$ dos casos têm a forma familiar (ELAf)). ${ }^{104}$ Sabe-se que $20 \%$ dos casos familiares desta doença são causados por mutações no gene que codifica a enzima antioxidante citosólica, $\mathrm{Cu}, \mathrm{Zn}$ superóxido dismutase (SOD1). ${ }^{102,105,106} \mathrm{~A}$ enzima $\mathrm{Cu}, \mathrm{Zn}$ superóxido dismutase (SOD1) é uma metaloproteína homodimérica de $32 \mathrm{KDa}$, citosólicas, tendo um $\mathrm{Cu}^{2+}$ e um $\mathrm{Zn}^{+}$. Mais de 190 mutações já são descritas, no entanto, os mecanismos moleculares da degeneração seletiva dos neurônios motores por SOD1 mutante em ELAf ainda são desconhecidos. A mutação resulta em um ganho de função tóxica, que alguns estudos sugerem poder estar relacionada ao efeito pró-oxidante da mutante SOD1 ${ }^{104}$ e /ou à formação de agregados citotóxicos de SOD1 ${ }^{104-106}$ Os eventos estruturais que levam à formação de oligômeros de alto peso molecular de SOD1 ainda são incertos. ${ }^{102,106}$ Alguns estudos sugerem que esses oligômeros seriam formados a partir de pontes dissulfeto entre os resíduos de cisteína livre presente na estrutura do dímero SOD1 (6 e Cys Cys 111). ${ }^{102}$ Estudo realizado por Kim e colaboradores mostrou que há indução da agregação de SOD1 na presença de ácidos graxos poli-insaturados. Esses agregados de alta massa molecular têm uma morfologia granular e apresentam citotoxicidade significativa. ${ }^{106}$

Elevados níveis de adutos de HHE-histidina também foram encontrados na medula espinhal de pacientes com ALS. ${ }^{107} \mathrm{O}$ HHE é um aldeído formado a partir da oxidação não enzimática do DHA e a detecção deste aduto indica que este ácido graxo é um alvo importante de oxidação na doença. Dado que o DHA é mais abundante que o AA no cérebro, também os neuroprostanos e neurofuranos podem se revelar biomarcadores atrativos. ${ }^{83}$ No entanto, até o momento não há trabalhos descrevendo a detecção destes produtos em ALS.

\section{CONSIDERAÇÕES FINAIS}

Em resumo, os trabalhos envolvendo DHA e doenças neurodegenerativas mostram que este ácido graxo pode desempenhar funções benéficas, inibindo a formação de agregados de peptídeos $\beta$-amiloides na doença de Alzheimer, quanto deletérias, como na doença de Parkison e ALS, onde este ácido graxo atua induzindo a formação de agregados proteicos. Além disso, o metabolismo oxidativo do DHA gera uma série de produtos, entre os quais se podem citar produtos com atividade anti-inflamatória e neuroprotetora, como as neuroprotectinas e resolvinas (formadas por vias enzimáticas de oxidação) e, também, produtos de oxidação reativos, como aldeídos (formados por oxidação não enzimática) os quais são capazes de danificar biomoléculas importantes como proteínas e ácidos nucleicos. Deste modo, pode-se concluir que estudos mais detalhados sobre o metabolismo e função do DHA e de seus produtos de oxidação são necessários para esclarecer o papel deste ácido graxo no cérebro, em particular, no desenvolvimento de doenças neurodegenerativas.

\section{AGRADECIMENTOS}

À FAPESP, CNPq, CAPES, INCT de Processos Redox em Biomedicina - Redoxoma e Pró-Reitoria de Pesquisa-USP pelo apoio financeiro e pelas bolsas concedidas.

\section{REFERÊNCIAS}

1. Larsson, S. C.; Kumlin, M.; Ingelman-Sundberg, M.; Wolk, A.; Am. J. Clin. Nutr. 2004, 79, 935.

2. Montine, T. J.; Morrow, J. D.; Am. J. Pathol. 2005, 166, 1283.

3. Lusis, A. J.; Nature 2000, 407, 233.

4. Chisolm, G. M.; Steinberg, D.; Free Radical Biol. Med. 2000, 28, 1815.

5. Nelson, D. L.; Cox, M. M.; Lehninger: Princípios de Bioquímica, $3^{\mathrm{a}}$ ed., Sarvier: São Paulo, 2002.

6. IUPAC-IUB; J. Lipid Res. 1978, 19, 114. 
7. Schmitz, G.; Ecker, J.; Progr. Lipid Res. 2008, 47, 147.

8. Simopoulos, A. P.; Biomed. Pharmacother. 2002, 56, 365.

9. Holman, R. T.; J. Am. Coll. Nutr. 1986, 5, 183.

10. Horrocks, L. A.; Farooqui, A. A.; Prostaglandins Leukot. Essent. Fatty Acids 2004, 70, 361.

11. Lauritzen, L.; Hansen, H. S.; Jørgensen, M. H.; Michaelsen, K. F.; Progr. Lipid Res. 2001, 40, 1.

12. Kim, H.-Y.; J. Biol. Chem. 2007, 282, 18661.

13. Salem, N.; Litman, B.; Kim, H. Y.; Gawrisch, K.; Lipids 2001, $36,945$.

14. Farooqui, A.; Horrocks, L.; Farooqui, T.; J. Mol. Neurosci. 2000, 14, 123.

15. Farooqui, A. A.; Horrocks, L. A.; Farooqui, T.; Chem. Phys. Lipids 2000, 106,1 .

16. Alessandri, J. M.; Poumes-Ballihaut, C.; Langelier, B.; Perruchot, M. H.; Raguenez, G.; Lavialle, M.; Guesnet, P.; Am. J. Clin. Nutr. 2003, 78, 702.

17. Gamoh, S.; Hashimoto, M.; Sugioka, K.; Shahdat Hossain, M.; Hata, N.; Misawa, Y.; Masumura, S.; Neuroscience 1999, 93, 237.

18. McGahon, B. M.; Martin, D. S. D.; Horrobin, D. F.; Lynch, M. A.; Neuroscience 1999, 94, 305.

19. Gordon, W. C.; Bazan, N. G.; J. Neurosci. 1990, 10, 2190.

20. Mirnikjoo, B.; Brown, S. E.; Kim, H. F. S.; Marangell, L. B.; Sweatt, J. D.; Weeber, E. J.; J. Biol. Chem. 2001, 276, 10888.

21. Rodriguez de Turco, E. B.; Belayev, L.; Liu, Y.; Busto, R.; Parkins, N.; Bazan, N. G.; Ginsberg, M. D.; J. Neurochem. 2002, 83, 515.

22. Kim, H.-Y.; Akbar, M.; Lau, A.; Edsall, L.; J. Biol. Chem. 2000, 275, 35215 .

23. Sprecher, H.; Biochim. Biophys. Acta (BBA) - Mol. Cell Biol. Lipid 2000, 1486, 219.

24. Mead, J. F.; Prog. Chem. Fats Other Lipids 1971, 9, 159.

25. Klenk, E.; Mohrhauer, H.; Hoppe-Seyler's Z. Physiol. Chem. 1960, 320, 218 .

26. Uauy, R.; Castillo, C.; J. Nutr. 2003, 133, 2962S.

27. Ayala, S.; Gaspar, G.; Brenner, R. R.; Peluffo, R. O.; Kunau, W.; J. Lipid Res. 1973, 14, 296.

28. Scott, B. L.; Bazan, N. G.; Proc. Natl. Acad. Sci. U. S. A. 1989, 86, 2903.

29. Voss, A.; Reinhart, M.; Sankarappa, S.; Sprecher, H.; J. Biol. Chem. 1991, 266, 19995.

30. Plourde, M.; Cunnane, S. C.; Appl. Physiol. Nutr. Metab. 2007, 32, 619.

31. Moore, S. A.; Yoder, E.; Murphy, S.; Dutton, G. R.; Spector, A. A.; J. Neurochem. 1991, 56, 518.

32. Williard, D. E.; Harmon, S. D.; Kaduce, T. L.; Spector, A. A.; Prostaglandins Leukot Essent Fatty Acids 2002, 67, 99.

33. Nakamura, M. T.; Nara, T. Y.; Annu. Rev. Nutr. 2004, 24, 345.

34. Aki, T.; Shimada, Y.; Inagaki, K.; Higashimoto, H.; Kawamoto, S.;Shigeta, S.;Ono, K.; Suzuki, O.; Biochem. Biophys. Res. Commun 1999, $255,575$.

35. Ferdinandusse, S.; Denis, S.; Mooijer, P. A. W.; Zhang, Z. Y.; Reddy, J. K.; Spector, A. A.; Wanders, R. J. A.; J. Lipid Res. 2001, 42, 1987.

36. Sprecher, H.; Luthria, D. L.; Mohammed, B. S.; Baykousheva, S. P.; J. Lipid Res. 1995, 36, 2471.

37. Rapoport, S. I.; J. Pediatr. 2003, 143, 26.

38. Rapoport, S. I.; Neurochem. Res. 1999, 24, 1403

39. Rapoport, S. I.; Chang, M. C.; Spector, A. A.; J. Lipid Res. 2001, 42, 678.

40. Breckenridge, W. C.; Morgan, I. G.; Zanetta, J. P.; Vincendon, G.; Biochim. Biophys. Acta 1973, 320, 681

41. Garcia, M. C.; Ward, G.; Ma, Y. C.; Salem, N.; Kim, H. Y.; J. Neurochem. 1998, 70, 24

42. Kalant, D.; Cianflone, K.; Curr. Opin. Lipidol. 2004, 15, 309.

43. Holub, B. J.; J. Biol. Chem. 1978, 253, 691.

44. Marszalek, J. R.; Lodish, H. F.; Annu. Rev. Cell Dev. Biol. 2005, 21, 633.
45. Lajtha, A.; Gibson, G.; Dienel, G.; Purdon, A.; Rapoport, S. Em Handbook of Neurochemistry and Molecular Neurobiology; Lajtha, G. E.; Gibson, G. A. D., eds.; Springer US: New York, 2007, chap. 4.6.

46. Chang, M.; Bell, J.; Purdon, A. D.; Chikhale, E.; Grange, E.; Neurochem. Res. 1999, 24, 399.

47. Rapoport, S. I.; Rao, J. S.; Igarashi, M.; Prostaglandins Leukot. Essent. Fatty Acids 2007, 77, 251.

48. Phillis, J. W.; Horrocks, L. A.; Farooqui, A. A.; Brain Res. Rev. 2006, 52,201

49. Kasuga, K.; Yang, R.; Porter, T. F.; Agrawal, N.; Petasis, N. A.; Irimia, D.;Toner, M.; Serhan, C. N.; J. Immunol. 2008, 181, 8677.

50. Serhan, C.; Arita, M.; Hong, S.; Gotlinger, K.; Lipids 2004, 39, 1125.

51. Serhan, C. N.; Hong, S.; Gronert, K.; Colgan, S. P.; Devchand, P. R.; Mirick, G.; Moussignac, R.-L.; J. Exp. Med. 2002, 196, 1025.

52. Schilstra, M. J.; Veldink, G. A.; Verhagen, J.; Vliegenthart, J. F.; Biochemistry 1992, 31, 7692.

53. Pomponi, M. F. L.; Gambassi, G.; Pomponi, M.; Di Gioia, A.; Masullo, C.; Ageing Res. Rev. 2011, 10, 124.

54. Serhan, C. N.; Curr. Opin. Clin. Nutr. Metab. Care 2005, 8, 115

55. Picq, M.; Chen, P.; Perez, M.; Michaud, M.; Véricel, E.; Guichardant, M.; Lagarde, M.; Mol. Neurobiol. 2010, 42, 48.

56. Hong, S.; Gronert, K.; Devchand, P. R.; Moussignac, R.-L.; Serhan, C. N.; J. Biol. Chem. 2003, 278, 14677.

57. Lukiw, W. J.; Cui, J. G.; Marcheselli, V. L.; Bodker, M.; Botkjaer, A.; Gotlinger, K.; Serhan, C. N.; Bazan, N. G.; J. Clin. Invest. 2005, 115, 2774

58. Pan, X.; Kaneko, H.; Ushio, H.; Ohshima, T.; Eur. J. Lipid Sci. Technol. 2005, 107, 228 .

59. Lyberg, A. M.; Adlercreutz, P.; Lipids 2006, 41, 67.

60. Simonian, N. A.; Coyle, J. T.; Annu. Rev. Pharmacol. Toxicol. 1996, 36, 83.

61. Yin, H.; Musiek, E. S.; Gao, L.; Porter, N. A.; Morrow, J. D.; J. Biol. Chem. 2005, 280, 26600

62. Girotti, A. W.; J. Lipid Res. 1998, 39, 1529.

63. Halliwell, B.; Gutteridge, J.; Free Radicals in Biology and Medicine, $3^{\text {rd }}$ ed, Oxford University Press: New York, 1999.

64. Frankel, E. N.; J. Sci. Food Agric. 1991, 54, 495

65. Porter, N. A.; Caldwell, S. E.; Mills, K. A.; Lipids 1995, 30, 277.

66. Miyamoto, S.; Martinez, G. R.; Medeiros, M. H. G.; Di Mascio, P.; J. Am. Chem. Soc. 2003, 125, 6172

67. Terao, J.; Matsushita, S.; Agric. Biol. Chem. 1981, 45, 587.

68. Terao, J.; Matsushita, S.; J. Am. Oil Chem. Soc. 1977, 54, 234.

69. Frankel, E. N.; Progr. Lipid Res. 1984, 23, 197.

70. Song, W.-L.; Lawson, J. A.; Reilly, D.; Rokach, J.; Chang, C.-T.; Giasson, B.; FitzGerald, G. A.; J. Biol. Chem. 2007, 283, 6

71. Bernoud-Hubac, N.; Davies, S. S.; Boutaud, O.; Montine, T. J.; Roberts, L. J., II; J. Biol. Chem. 2001, 276, 30964.

72. Kawai, Y.; Takeda, S.; Sugita, S.; Takahara, K.; Terao, J.; Free Radical Biol. Med. 2004, 37, S-109.

73. Kawai, Y.; Fujii, H.; Okada, M.; Tsuchie, Y.; Uchida, K.; Osawa, T.; J. Lipid Res. 2006, 47, 1386.

74. Roberts Ii, L. J.; Montine, T. J.; Markesbery, W. R.; Tapper, A. R.; Hardy, P.; Chemtob, S.; Dettbarn, W. D.; Morrow, J. D.; J. Biol. Chem. 1998, 273, 13605.

75. Reich, E. E.; Zackert, W. E.; Brame, C. J.; Chen, Y.; Roberts, L. J.; Hachey, D. L.; Montine, T. J.; Morrow, J. D.; Biochemistry 2000, 39, 2376.

76. Arneson, K. O.; Roberts Ii, L. J.; Brown, H. A.; Methods of Enzymology 2007, 433, 127

77. Esterbauer, H.; Schaur, R. J.; Zollner, H.; Free Radical Biol. Med. 1991, 11,81 .

78. de Zwart, L. L.; Meerman, J. H. N.; Commandeur, J. N. M.; Vermeulen, N. P. E.; Free Radical Biol. Med. 1999, 26, 202. 
79. Ross, C. A.; Poirier, M. A.; Nat. Med. 2004, 10 Suppl, S10.

80. Jomova, K.; Vondrakova, D.; Lawson, M.; Valko, M.; Molecular Cell Biochemistry 2010, 345, 91.

81. Zhao, Y.; Calon, F.; Julien, C.; Winkler, J. W.; Petasis, N. A.; Lukiw, W. J.; Bazan, N. G.; PLoS One 2011, 6, e15816.

82. Soderberg, M.; Edlund, C.; Kristensson, K.; Dallner, G.; Lipids 1991, $26,421$.

83. Nourooz-Zadeh, J.; Liu, E. H. C.; Yhlen, B.; Anggard, E. E.; Halliwell, B.; J. Neurochem. 1999, 72, 734.

84. Montine, T. J.; Neely, M. D.; Quinn, J. F.; Beal, M. F.; Markesbery, W. R.; Roberts, L. J.; Morrow, J. D.; Free Radical Biol. Med. 2002, 33, 620.

85. Barnham, K. J.; Masters, C. L.; Bush, A. I.; Nat. Rev. Drug. Discov. 2004, 3, 205 .

86. Arneson, K. O.; Roberts, L. J.; Lipidomics and Bioactive Lipids 2007, $433,127$.

87. Forman, H. J.; Dickinson, D. A.; Free Radical Biol. Med. 2004, 37, 594.

88. Davies, S. S.; Amarnath, V.; Montine, K. S.; Bernoud-Hubac, N.; Boutaud, O.; Montine, T. J.; Roberts, L. J., II; FASEB J. 2002, 16, 715.

89. Jicha, G. A.; Markesbery, W. R.; Clin. Interv. Aging 2010, 5, 45.

90. Grimm, M. O. W.; Kuchenbecker, J.; Groesgen, S.; Burg, V. K.; Hundsdoerfer, B.; Rothhaar, T. L.; Friess, P.; de Wilde, M. C.; Broersen, L. M.; Penke, B.; Peter, M.; Vigh, L.; Grimm, H. S.; Hartmann, T.; J. Biol. Chem. 2011, 286, 14028.

91. Cole, S. L.; Vassar, R.; Curr. Alzheimer Res. 2008, 5, 100.

92. Willem, M.; Lammich, S.; Haass, C.; Semin. Cell Dev. Biol. 2009, 20, 175.

93. Lukiw, W. J.; Bazan, N. G.; J. Nutr. 2008, 138, 2510.

94. Oksman, M.; Iivonen, H.; Hogyes, E.; Amtul, Z.; Penke, B.; Leenders, I.; Broersen, L.; Lutjohann, D.; Hartmann, T.; Tanila, H.; Neurobiol. Dis. 2006, 23, 563 .
95. Broersen, K.; van den Brink, D.; Fraser, G.; Goedert, M.; Davletov, B.; Biochemistry 2006, 45, 15610.

96. Karube, H.; Sakamoto, M.; Arawaka, S.; Hara, S.; Sato, H.; Ren, C. H.; Goto, S.; Koyama, S.; Wada, M.; Kawanami, T.; Kurita, K.; Kato, T.; FEBS Lett. 2008, 582, 3693.

97. Spillantini, M. G.; Schmidt, M. L.; Lee, V. M.; Trojanowski, J. Q.; Jakes, R.; Goedert, M.; Nature 1997, 388, 839.

98. De Franceschi, G.; Frare, E.; Bubacco, L.; Mammi, S.; Fontana, A.; de Laureto, P. P.; J. Mol. Biol. 2009, 394, 94.

99. Israeli, E.; Sharon, R.; J. Neurochem. 2009, 108, 465.

100. Sharon, R.; Bar-Joseph, I.; Mirick, G. E.; Serhan, C. N.; Selkoe, D. J.; J. Biol. Chem. 2003, 278, 49874.

101. Chinta, S. J.; Andersen, J. K.; Biochim Biophys Acta 2008, 1780, 1362.

102. Banci, L.; Bertini, I.; Boca, M.; Girotto, S.; Martinelli, M.; Valentine, J. S.; Vieru, M.; PLoS One 2008, 3, e1677.

103. Bendotti, C.; Carri, M. T.; Antioxid. Redox Signal 2009, 11, 1519.

104. Rakhit, R.; Cunningham, P.; Furtos-Matei, A.; Dahan, S.; Qi, X. F.; Crow, J. P.; Cashman, N. R.; Kondejewski, L. H.; Chakrabartty, A.; J. Biol. Chem. 2002, 277, 47551.

105. Valentine, J. S.; Doucette, P. A.; Zittin Potter, S.; Annu. Rev. Biochem. 2005, 74, 563.

106. Kim, Y. J.; Nakatomi, R.; Akagi, T.; Hashikawa, T.; Takahashi, R.; J. Biol. Chem. 2005, 280, 21515.

107. Shibata, N.; Yamada, S.; Uchida, K.; Hirano, A.; Sakoda, S.; Fujimura, H.; Sasaki, S.; Iwata, M.; Toi, S.; Kawaguchi, M.; Yamamoto, T.; Kobayashi, M.; Brain Res. Rev. 2004, 1019, 170. 